\title{
Potential of stem-cell-based therapies for liver regeneration
}

\author{
Yunfang Wang ${ }^{1}$, Junnian Zhou ${ }^{1}$, Haiyun $\mathrm{Pei}^{1}$, Yue Teng ${ }^{1}$, Min Wang ${ }^{1}$, Yali Jia ${ }^{1}$, Xuetao Pei ${ }^{1}$

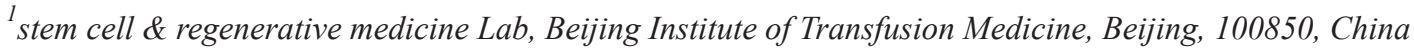

Because of the persistent donor shortage, several stem cell based therapies for liver disease have been proposed; namely, extracorporeal bioartificial liver devices, cell transplantation, and tissue-engineered constructs. Despite their differences, these therapies share a requirement for adequate cell supply and stability of liver-specific functions. Although some extracorporeal cell-based support devices are already in clinical trials, the efficacy of engineered cell therapies in the management of liver disease can be improved further by incorporating strategies for maximizing stem cell derived hepatocyte function. Current cell-based approaches will rely on a variety of cell sources, whether primary or stem cells, which will ultimately interact with the microenvironment en route to providing key liverspecific functions. To apply such strategies, lessons must be drawn from classic liver biology as well as the experience of transplant medicine in supporting liver failure patients.

Our research is focusing on bone marrow or embryonic stem cell derived hepatocytes and the interaction with their microenvironment and develops microfabrication tools to improve cellular therapies for liver disease, especially on cell-cell, cell- extracellular matrices (ECMs) interactions on determination of stem cell differentiation and the mechanism. Coculture system and 3D organoid culture in bioreactor have been used to maximize hepatocyte function, facilitate design of effective cellular therapies for liver disease, and improve fundamental understanding of liver physiology and pathophysiology. Then, we constructed implantable bioartificial liver assist system with the stem cell derived hepatocytes as the seed cells by means of biofabrication, and explored effects of biochemical and mechanic characters on hepatic modality, structure and function. We are also interested in using arrays of hepatic oval cells as high-throughput platforms to study fundamental aspects of stem cell biology (Cell-Based BioMEMS) using a diverse repertoire of tools. By co-immuno- precipitation and micropattern, we propose a new hypothesis that epithelial morphogeny (EPM) is involved in differentiation of hepatic stem/ progenitor cells through EPMcytoskeleton CSK (-Mitosis spindle orientation MSO) system which mediates biochemical-mechanical signals.

Keywords: stem cell, hepatocyte, bioartificial liver, regenerative medicine

Cell Research (2008) 18:s11. doi: 10.1038/cr.2008.101; published online 4 August 2008

Correspondence: Xuetao Pei

E-mail: peixt@nic.bmi.ac.cn

Xuetao PEI, PhD, Director, Professor of Beijing Institute of Transfusion Medicine, Chief Scientist, Key Project of State High-tech Project (863 Program) "stem Cell and Tissue Engineering for Regenerative Medicine", working on Stem Cell Biology and Regenerative Medicine. Have edited and published more than 9 literatures as the editor-in-chief and also published more than 200 papers in the Journals such as Stem Cells, JBC, JCP, Apoptosis, Liver Transplantation, Exp Hematol, Exp Neurol, etc. 40 patents have been applied for, and 9 patents were authorized. 